\title{
The localization of rice prolamin species in protein body type $I$ is determined by the temporal control of gene expression of the respective prolamin promoters
}

\author{
Ai Sasou, ${ }^{1, a}$, Takanari Shigemitsu' ${ }^{1}$, Shigeto Morita ${ }^{1,2}$, Takehiro Masumura ${ }^{1,2, *}$ \\ ${ }^{1}$ Laboratory of Genetic Engineering, Graduate School of Life and Environmental Sciences, Kyoto Prefectural University, \\ Shimogamo, Kyoto 606-8522, Japan; ${ }^{2}$ Biotechnology Research Department, Kyoto Prefectural Agriculture, Forestry and \\ Fisheries Technology Research Center, Kitainayazuma, Seika-cho, Soraku-gun, Kyoto 619-0244, Japan \\ *E-mail: masumura@kpu.ac.jp Tel \& Fax: +81-75-703-5675
}

Received May 26, 2018; accepted September 18, 2018 (Edited by K. Hiratsuka)

\begin{abstract}
Rice prolamin species form a layered structure in the protein body type I (PB-I) storage organelle. Rice prolamins are classified as $10 \mathrm{kDa}, 13 \mathrm{a}-1,13 \mathrm{a}-2,13 \mathrm{~b}-1,13 \mathrm{~b}-2$ and $16 \mathrm{kDa}$ prolamin. Prolamin species form layer structure in PB-I in order of $10 \mathrm{kDa}$ core, $13 \mathrm{~b}-1$ layer, $13 \mathrm{a}$ (13a-1 and 13a-2) and $16 \mathrm{kDa}$ middle layer and 13b-2 outer-most layer. In a previous study, we showed that the fusion proteins in 13b-2 prolamin-GFP, 13a-1 prolamin-GFP and $10 \mathrm{kDa}$ prolamin-GFP were localized in the same layer of PB-I as the native prolamin, when they were expressed by their respective native prolamin promoters. Our preliminary study suggested that the temporal control of the native prolamin promoters was responsible for the localization of the respective prolamins. The aim of this study was to determine whether the use of a prolamin promoter other than the native prolamin promoter would change the localization of prolamin-GFP fusion proteins. For this purpose, we generated transgenic lines expressing 13b-2 prolamin-GFP and 13a-1 prolamin-GFP fusion proteins driven by each prolamin promoter other than the native prolamin promoter. As a result, the localization of the fusion protein in PB-I was changed. Based on our results, foreign protein localization in PB-I can be achieved by the temporal control of the different prolamin promoters.
\end{abstract}

Key words: green fluorescent protein, layer structure, prolamin, prolamin promoter, protein body type I, rice.

Rice prolamins, alcohol-soluble proteins in the rice seed, are synthesized in the endoplasmic reticulum (ER) and form protein body type I (PB-I) in endosperm cells (Tanaka et al. 1980; Yamagata and Tanaka 1986; Yamagata et al. 1982). Rice prolamins are classified by size as $10 \mathrm{kDa}, 13 \mathrm{kDa}$ and $16 \mathrm{kDa}$ (Masumura et al. 1989; Mitsukawa et al. 1999a, b). In addition, $13 \mathrm{kDa}$ prolamins are subdivided into Cys-rich 13a prolamin (13a-1 and 13a-2) and Cys-less 13b prolamin (13b-1 and 13b-2) (Mitsukawa et al. 1999a; Saito et al. 2012). Previously, we reported that these prolamin species form a layered structure in PB-Is, with the $10 \mathrm{kDa}$ prolamin at the core of PB-I, the 13b-1 prolamin in the inner layer surrounding the $10 \mathrm{kDa}$ prolamin core, $16 \mathrm{kDa}$ and $13 \mathrm{a}$ prolamin in the middle layer, and $13 \mathrm{~b}-2$ prolamin in the outer-most layer of PB-I (Saito et al. 2012).

The formation of a layered structure in PB-Is was suggested to be dependent on the gene expression patterns of the respective prolamin species in rice seeds. We previously demonstrated that during development of rice seeds, the $10 \mathrm{kDa}$ prolamin gene was expressed first, followed shortly by the 13b-1 prolamin gene, then the $16 \mathrm{kDa}$ and $13 \mathrm{a}$ prolamin genes, and finally the 13b-2 prolamin gene (Saito et al. 2012). The location of the various respective prolamin polypeptides in PB-I coincided with the temporal expression patterns of their respective genes. We previously found that when the 13b-2 prolamin-GFP, 13a-1 prolamin-GFP and $10 \mathrm{kDa}$ prolamin-GFP fusion proteins were expressed by their native promoters, each prolamin-GFP fusion protein was localized in the same layer in PB-Is as the native prolamin (Saito et al. 2012; Sasou et al. 2016). Based on these previous studies, the control of the respective prolamin promoters appears to be important for the localization of prolamin in PB-I.

Therefore, in this study, to investigate in more detail the relationship between the temporal control of prolamin promoters and the localization of prolamin species in PB-Is, we generated and analyzed transgenic rice seeds expressing 13b-2 prolamin-GFP fusion

Abbreviations: PB-I, protein body type I; GFP, green fluorescent protein; ER, endoplasmic reticulum.

${ }^{a}$ Present address: Division of Mucosal Immunology, the Institute of Medical Science, The University of Tokyo, 4-6-1 Shirokanedai, Minato-ku, Tokyo 108-8639 Japan.

This article can be found at http://www.jspcmb.jp/

Published online December 14, 2018 
proteins under the control of the 13a-1 prolamin and $10 \mathrm{kDa}$ prolamin promoters, and expressing 13a-1 prolamin-GFP fusion proteins under the control of the $13 \mathrm{~b}-2$ prolamin and $10 \mathrm{kDa}$ prolamin promoter, respectively (Figure 1).

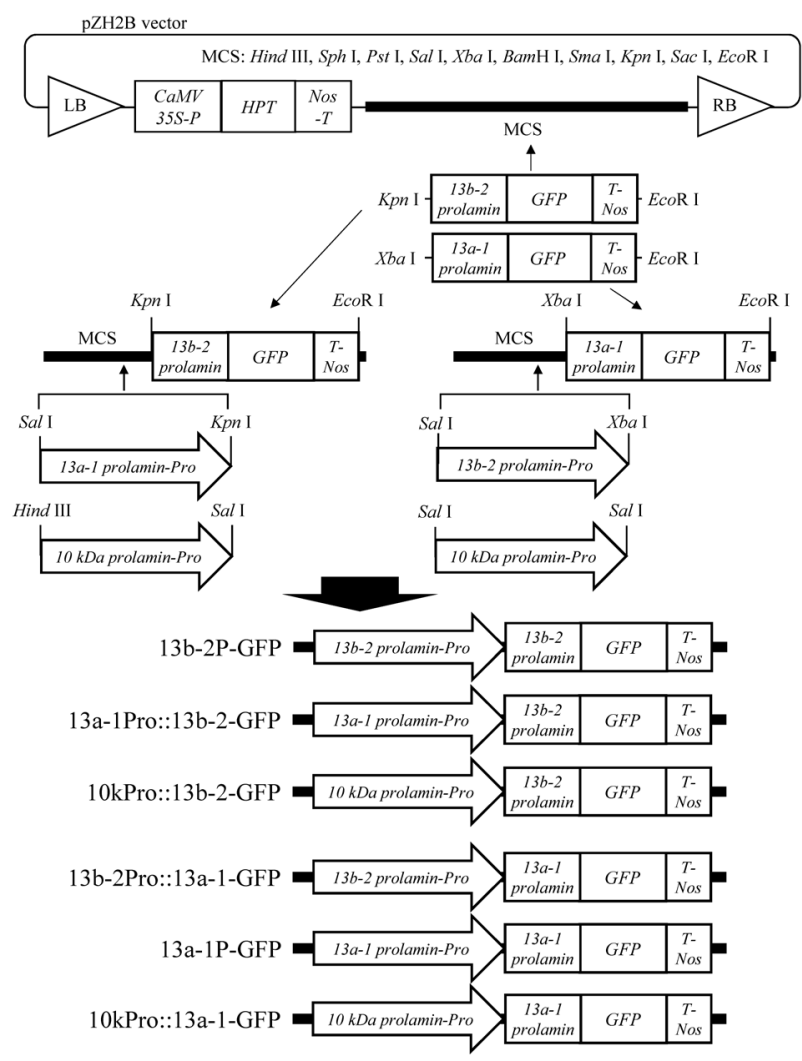

Figure 1. The flow diagram of constructions expressing 13b-2 prolamin-GFP fusion protein and 13a-1 prolamin-GFP fusion protein under the control of various prolamin promoters. 13b-2P-GFP and 13a-1P-GFP are transgenic lines expressing 13b-2 prolamin-GFP and 13a-1 prolamin-GFP fusion prolamin respectively, which were generated previously (Sasou et al. 2016). MCS, multiple cloning site; CaMV35S-P, cauliflower mosaic virus $35 \mathrm{~S}$ promoter; HPT, hygromycin phosphotransferase; Nos-T, nopaline synthase terminator; 13b-2 prolamin-Pro, 13b-2 prolamin promoter; 13a-1 prolamin-Pro, 13a-1 prolamin promoter; $10 \mathrm{kDa}$ prolamin-Pro, $10 \mathrm{kDa}$ prolamin promoter; GFP, green fluorescent protein.
Constructions expressing 13b-2 prolamin-GFP fusion protein and 13a-1 prolamin-GFP fusion protein driven by the respective native promoters were generated previously (Sasou et al. 2016). Constructions expressing 13b-2 prolamin-GFP fusion protein and 13a-1 prolaminGFP fusion protein driven by other, non-native prolamin promoters were generated based on the $\mathrm{pZH} 2 \mathrm{~B}$ vector (Kuroda et al. 2010). Respective sequences of the promoter region (1500 bp from transcriptional initiation point) were amplified from genomic DNA. The coding regions for 13b-2 prolamin ( $\lambda R M 4), 13 \mathrm{a}-1$ prolamin $(\lambda R M 1)$ and $10 \mathrm{kDa}$ prolamin $(\lambda R P 10)$ were amplified from the 13b-2P-GFP, 13a-1P-GFP and 10kP-GFP vectors constructed previously (Sasou et al. 2016).

The DNA fragments of 13b-2 prolamin-GFP-NOS (containing KpnI and EcoRI site) and 13a-1 prolaminGFP-NOS (containing XbaI and EcoRI site) were amplified from 13b-2P-GFP and 13a-1P-GFP (Sasou et al. 2016) as the template using the primer set shown in Table 1. PCR fragments were digested with KpnI/XbaI and $E c o$ RI, and inserted in the multiple cloning site of a $\mathrm{pZH} 2 \mathrm{~B}$ vector (Figure 1). The promoter regions of the 13b-2 prolamin, 13a-1 prolamin and $10 \mathrm{kDa}$ prolamin were also amplified from 13b-2P-GFP, 13a-1P-GFP and $10 \mathrm{kP}-\mathrm{GFP}$ (Sasou et al. 2016) using the primer set in Table 1. PCR fragments of the $13 \mathrm{~b}-2$ prolamin promoter (containing the SalI and XbaI sites) and 13a1 prolamin promoter (containing the SalI and KpnI sites) were digested with an adequate combination of restriction endonucleases and inserted in the upstream of the each prolamin-GFP-NOS sequence in the pZH2B vector (Figure 1). The PCR fragment of $10 \mathrm{kDa}$ prolamin promoter (containing the HindIII and SalI sites) was digested with an adequate combination restriction endonucleases and inserted in the upstream of the 13b2 prolamin-GFP in pZH2B vector (Figure 1). Besides, The PCR fragment of $10 \mathrm{kDa}$ prolamin promoter (containing the SalI and SalI sites) was also digested with salI and inserted the upstream of 13a-1 prolaminGFP in PZH2B vector because there is the HindIII site in the cording region of 13a-1 prolamin (Figure 1). The

Table 1. Primer sequences used to generate the constructs expressing the prolamin-GFP fusion proteins under the control of other prolamin promoters.

\begin{tabular}{|c|c|}
\hline Primer name & Upstream and downstream primer sequences \\
\hline 13b-2-GFP T-Nos fw $+K p n \mathrm{I}$ & $5^{\prime}$-aggggtaccgcattatacagcaaaataga-3' \\
\hline 13a-1-GFP T-Nos fw $+X b a \mathrm{I}$ & $5^{\prime}$-atatctagagttgaagcatagtagtagaatcc- $3^{\prime}$ \\
\hline T-Nos rv+EcoRI & $5^{\prime}$-agagaattcccgatctagtaacatagatg- $3^{\prime}$ \\
\hline 13b- 2 prolamin promoter $\mathrm{fw}+$ SalI & $5^{\prime}$-gtcgactgtccatcattcctaacaagagg- $3^{\prime}$ \\
\hline $13 \mathrm{~b}-2$ prolamin promoter $\mathrm{rv}+\mathrm{Xba \textrm {I }}$ & $5^{\prime}$-atatctagactgtgtgaacgatgaggagg- $3^{\prime}$ \\
\hline 13a-1 prolamin promoter $\mathrm{fw}+$ SalI & $5^{\prime}$-aaagtcgacgaggagggtcagctgtggc- $3^{\prime}$ \\
\hline 13a-1 prolamin promoter $\mathrm{rv}+K p n \mathrm{I}$ & $5^{\prime}$-ataggtacctgtgtgaaggatgaggaggg- $-3^{\prime}$ \\
\hline $10 \mathrm{kDa}$ prolamin promoter $\mathrm{fw}+$ HindIII & $5^{\prime}$-ataaagcttgacgggatcacgatctggg- $3^{\prime}$ \\
\hline $10 \mathrm{kDa}$ prolamin promoter $\mathrm{fw}+$ SalI & $5^{\prime}$-atagtcgacgacgggatcacgatctggg- $3^{\prime}$ \\
\hline $10 \mathrm{kDa}$ prolamin promoter $\mathrm{rv}+$ SalI & $5^{\prime}$-atagtcgacggaataatggttgcctaggg- $3^{\prime}$ \\
\hline
\end{tabular}


orientation of $10 \mathrm{kDa}$ prolamin promoter (containing the SalI and SalI sites) was confirmed using the forward primer which binds to the interior of $10 \mathrm{kDa}$ prolamin promoter (5'-CGG TCT AGA TGA CTA GAT TG-3') and the reverse primer which binds to the interior of GFP (5'-GTA GGT GAA GGT GGT CAC GA-3') by PCR, after then confirmed by sequence analysis. We named the resulting binary vectors $13 \mathrm{a}-1$ Pro::13b-2GFP, 10kPro::13b-2-GFP, 13b-2Pro::13a-1-GFP, and 10kPro::13a-1-GFP (Figure 1). These binary vectors were introduced into rice calli using an Agrobacteriummediated method (Hiei et al. 1994). The rice calli containing the transgene were selected by hygromycin B (Nacalai Tesque, Kyoto, Japan) and transferred to redifferentiation medium. Then, the shoots from selected calli were transferred to plant pots containing soil in a naturally illuminated temperature-controlled $\left(28^{\circ} \mathrm{C}\right)$ greenhouse of the Biotechnology Research Department, Kyoto Prefectural Agriculture, Forestry, and Fisheries Technology Research Center, Japan.

To clarify the localization of prolamin-GFP fusion proteins in each transgenic rice line, the observation of frozen sections prepared with mature transgenic rice seeds was performed by confocal laser microscopic analysis. The fluorescent observation was performed as previously described (Saito et al. 2008; Sasou et al. 2016). Briefly, frozen sections (5- $\mu \mathrm{m}$ thick) of each transgenic rice seed were prepared by the method developed by Saito et al. (2008), and were stained with $10-\mathrm{nM}$ rhodamine $\mathrm{B}$, which is commonly used to stain the peripheral region of $\mathrm{PB}-\mathrm{Is}$, for $10 \mathrm{~min}$ at room temperature. The sections stained with rhodamine B were observed using a confocal laser scanning microscope (A1 confocal imaging system; Nikon Instech, Tokyo).

In transgenic rice of the $13 \mathrm{~b}-2 \mathrm{P}-\mathrm{GFP}$ line, the $13 \mathrm{~b}-2$ prolamin-GFP fusion protein was observed in the outermost layer of PB-Is (Figure 2A-C). In the 13a-1Pro::13b2-GFP line, the prolamin-GFP fusion proteins were observed in the middle layer of PB-I (Figure 2D-F). In the 10kPro::13b-2-GFP line, the prolamin-GFP fusion proteins were mainly observed in the core region of $\mathrm{PB}$ Is, as small spots, although a small number were also observed in the layer surrounding the core region of $\mathrm{PB}$ Is (Figure 2G-I). Although the same fusion protein was expressed in each transgenic rice line, the localization of the fusion protein was changed by the type of prolamin promoter.

Similar results were obtained with transgenic rice lines expressing the 13a-1 prolamin-GFP fusion protein. In the 13b-2Pro::13a-1-GFP line, the 13a-1 prolaminGFP fusion proteins were observed in the outer-most layer of PB-Is (Figure 3A-C). In the 13a-1P-GFP line, the GFP signal was observed as the ring shape in PB-I and the prolamin-GFP fusion proteins were seemed to
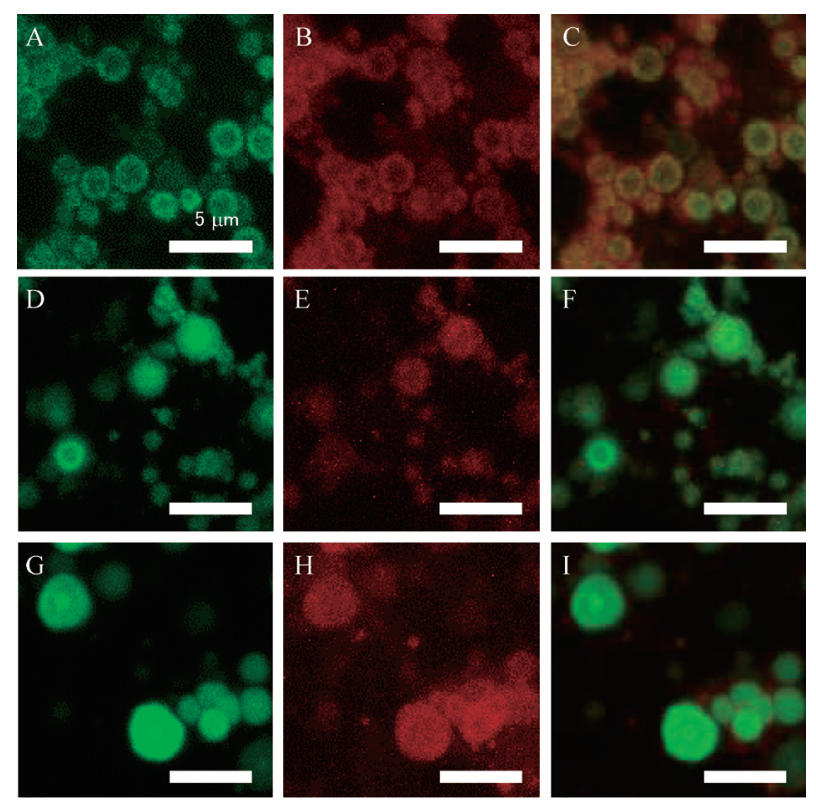

Figure 2. Localization of $13 \mathrm{~b}-2$ prolamin-GFP fusion proteins under the control of promoters derived from $13 \mathrm{~b}-2$ prolamin (A-C), 13a-1 prolamin $(\mathrm{D}-\mathrm{F})$ and $10 \mathrm{kDa}$ prolamin $(\mathrm{G}-\mathrm{I})$. Green indicates the GFP signal $(A, D, G)$, and red indicates the signal of rhodamine $B$, which stains the peripheral region of $\mathrm{PB}-\mathrm{Is}(\mathrm{B}, \mathrm{E}, \mathrm{H})$. C, F, I indicate the merged images. Bars $=5 \mu \mathrm{m}$.
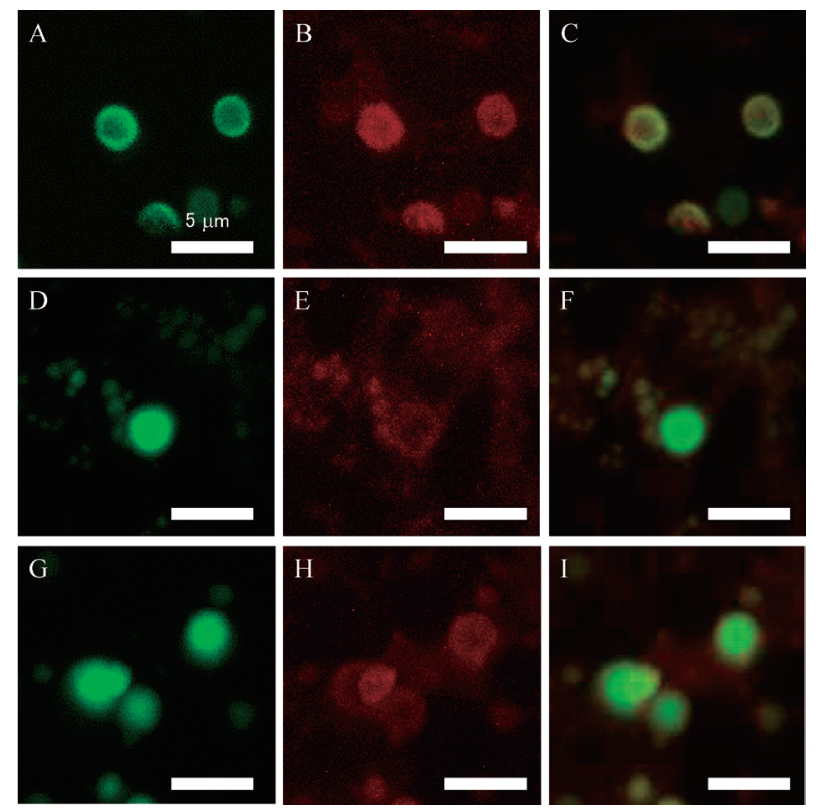

Figure 3. Localization of 13a-1 prolamin-GFP fusion proteins under the control of the promoter derived from 13b-2 prolamin (A-C), 13a-1 prolamin $(\mathrm{D}-\mathrm{F})$ and $10 \mathrm{kDa}$ prolamin $(\mathrm{G}-\mathrm{I})$. Green indicates the GFP signal $(A, D, G)$, and red indicates the signal of rhodamine $B$, which stains the peripheral region of $\mathrm{PB}-\mathrm{Is}(\mathrm{B}, \mathrm{E}, \mathrm{H})$. C, F, I indicate the merged images. Bars $=5 \mu \mathrm{m}$.

localize in the middle layer of PB-Is (Figure 3D-F). In the 10kPro::13a-1-GFP line, the GFP signal was observed as the small spots in PB-I and the prolamin-GFP fusion proteins were localized in the core region of PB-Is 
(Figure 3G-I).

The results of the fluorescence microscopic analysis thus suggested that the localization of the respective prolamin species was determined by the expression of the respective prolamin promoter, regardless of the type of prolamin polypeptides being expressed. We previously showed that the 13b-2 prolamin-GFP, 13a-1 prolamin-GFP and $10 \mathrm{kDa}$ prolamin-GFP fusion proteins expressed under the control of their native promoters were localized in the same layer as the respective endogenous prolamins in PB-I (Sasou et al. 2016). Our preliminary study suggested that the temporal control of the respective prolamin promoters determined the localization of each prolamin in its specific layer of PB-Is (Sasou et al. 2016). Moreover, we reported that the prolamin signal peptide and GFP fusion protein expressed by the native prolamin promoter were not localized in PB-Is, while the fusion proteins containing hydrophobic portion sequence in mature prolamin polypeptides was localized in PB-Is (Sasou et al. 2017). In this study, the localization of the $13 \mathrm{~b}-2$ prolaminGFP fusion protein and 13a-1 prolamin-GFP fusion protein were both regulated by the expression pattern of the each prolamin promoter. The results of this study suggested that the prolamin promoter is important for the localization of GFP-tagged prolamins in a particular layer of the PB-I.

Cys-rich 13a-1 prolamin is thought to be able to form disulfide bonds with other Cys-rich prolamin (e.g., $10 \mathrm{kDa}$ prolamin). However, $13 \mathrm{~b}-2$ prolamin is the Cys-less type prolamin, so $13 \mathrm{~b}-2$ prolamin may interact with other prolamin by hydrophobic interaction alone. 13a-1 Prolamin and 13b-2 prolamin may be accumulated in PB-I by different properties in interaction of other prolamin species, but it is difficult to perform experiments for interaction analysis such as co-immunoprecipitation (co-IP) because of the hydrophobicity of prolamin polypeptides in their entirety. Some reports have shown the possibility of analyzing the formation of $\mathrm{PBs}$ by expressing respective prolamin polypeptides in heterologous cells without other complicated factors (Masumura et al. 2015; Shigemitsu et al. 2013); hence, it may be possible to analyze the interaction between respective prolamin species by co-expressing some kind of prolamin polypeptides in the same transgenic rice calli or the yeasts.

We conclude altogether the previous report and this research as followed. The temporal control of the respective prolamin promoter is a key factor determining the localization of the respective prolamin polypeptides within the layered structure of the PB-I. The prolamin signal peptides were considered to help the fusion protein transfer into the ER for entering secretory pathway rather than for accumulating into PB-Is.
Then, mature prolamin polypeptides are thought to be important to retain prolamin-GFP fusion proteins in PB-Is.

Our finding in this study will provide a novel technique for the expression of useful proteins such as oral vaccine antigens in PB-Is because of their indigestibility (Sasou et al. 2016). If we can control the localization of a vaccine protein in a specific layer of PBIs by linking it with the appropriate prolamin promoter, it may be possible to develop techniques to encapsulate vaccines that can be released in the small intestine without undergoing exposure to the harsh environment of the stomach.

\section{Acknowledgements}

We are grateful to Dr. Masaharu Kuroda, National Agricultural Research Center, for providing the pZH2B binary vector. We would like to thank Mr. Kazuo Nakagawa and Mr. Toyofumi Kameoka of Nikon Instech, for supporting the capture of fluorescence images with a confocal laser scanning microscope. This work was supported by the JSPS KAKENHI, with a Grant-in-Aid for Scientific Research (C) (\#21580417) and Grant-in-Aid for Challenging Exploratory Research (\#15K14923) to T.M.

\section{Conflict of interest}

The authors have no conflicts of interest to declare.

\section{References}

Hiei Y, Ohta S, Komari T, Kumashiro T (1994) Efficient transformation of rice (Oryza sativa L.) mediated by Agrobacterium and sequence analysis of the boundaries of the T-DNA. Plant J 6: 271-282

Kuroda M, Kimizu M, Mikami C (2010) A simple set of plasmids for the production of transgenic plants. Biosci Biotechnol Biochem 74: 2348-2351

Masumura T, Shibata D, Hibino T, Kato T, Kawabe K, Takeda G, Tanaka K, Fujii S (1989) cDNA cloning of an mRNA encoding a sulfur-rich $10 \mathrm{kDa}$ prolamin polypeptide in rice seeds. Plant $\mathrm{Mol}$ Biol 12: 123-130

Masumura T, Shigemitsu T, Morita S, Satoh S (2015) Identification of the region of rice $13 \mathrm{kDa}$ prolamin essential for the formation of ER-derived protein bodies using a heterologous expression system. Biosci Biotechnol Biochem 79: 566-573

Mitsukawa N, Konishi R, Kidzu K, Ohtsuki K, Masumura T, Tanaka K (1999a) Amino acid sequencing and cDNA cloning of rice seed storage proteins, the $13 \mathrm{kDa}$ prolamins, extracted from type I protein bodies. Plant Biotechnol 16: 103-113

Mitsukawa N, Konishi R, Uchiki M, Masumura T, Tanaka K (1999b) Molecular cloning characterization of acysteine-rich $16.6-\mathrm{kDa}$ proalmin in rice seeds. Biosci Biotechnol Biochem 63: $1851-1858$

Saito Y, Nakatsuka N, Shigemitsu T, Tanaka K, Morita S, Satoh S, Masumura T (2008) Thin frozen film method for visualization of storage proteins in mature rice grains. Biosci Biotechnol Biochem 72: $2779-2781$

Saito Y, Shigemitsu T, Yamasaki R, Sasou A, Goto F, Kishida K, Kuroda M, Tanaka K, Morita S, Satoh S, et al. (2012) Formation mechanism of the internal structure of type I protein bodies 
in rice endosperm: Relationship between the localization of prolamin species and the expression of individual genes. Plant $J$ 70: 1043-1055

Sasou A, Shigemitsu T, Saito Y, Tanaka M, Morita S, Masumura T (2016) Control of foreign polypeptide accumulation in specific layers of protein body type I in rice seed. Plant Cell Rep 35: 1287-1295

Sasou A, Shigemitsu T, Morita S, Masumura T (2017) Accumulation of foreign polypeptides to rice seed protein body type I using prolamin portion sequences. Plant Cell Rep 36: 481-491

Shigemitsu T, Masumura T, Morita S, Satoh S (2013) Accumulation of rice prolamin-GFP fusion proteins induces ER-derived protein bodies in transgenic rice calli. Plant Cell Rep 32: 389-399

Tanaka K, Sugimoto T, Ogawa M, Kasai Z (1980) Isolation and characterization of two types of protein bodies in the rice endosperm. Agric Biol Chem 44: 1633-1639

Yamagata H, Sugimoto T, Tanaka K, Kasai Z (1982) Biosynthesis of storage proteins in developing rice seeds. Plant Physiol 70 : 1094-1100

Yamagata H, Tanaka K (1986) The site of synthesis and accumulation of rice storage proteins. Plant Cell Physiol 27: 135-145 